\title{
Le cadre matériel des cours de chimie dans l'enseignement supérieur à Paris au XIXe siècle
}

The material context of chemistry lessons in higher education in 19th century Paris

Die materiellen Rahmenbedingungen des Chemieunterrichts der Pariser Hochschulen im 19. Jahrhundert

El marco material de las clases de química en la enseñanza superior en París en el siglo XIX

\section{Sacha Tomic}

\section{CpenEdition}

\section{Journals}

Édition électronique

URL : https://journals.openedition.org/histoire-education/2326

DOI : $10.4000 /$ histoire-education.2326

ISSN : 2102-5452

Éditeur

ENS Éditions

\section{Édition imprimée}

Date de publication : 1 avril 2011

Pagination : $57-83$

ISBN : 978-2-84788-331-2

ISSN : 0221-6280

\section{Référence électronique}

Sacha Tomic, «Le cadre matériel des cours de chimie dans l'enseignement supérieur à Paris au XIXe siècle », Histoire de l'éducation [En ligne], 130 | 2011, mis en ligne le 01 avril 2013, consulté le 20 mai 2021. URL : http://journals.openedition.org/histoire-education/2326 ; DOI : https://doi.org/10.4000/ histoire-education.2326 


\title{
Le cadre matériel des cours de chimie dans l'enseignement supérieur à Paris au XIX ${ }^{\mathrm{e}}$ siècle
}

\author{
Sacha TOMIC
}

Longtemps associée à la médecine, à la pharmacie et aux autres arts (métallurgie, teinturerie, tannerie, etc.) ${ }^{1}$, la chimie s'est peu à peu émancipée de son rôle de science de service pour devenir au XIX" siècle une "science de professeurs $"^{2}$. La chimie post-lavoisienne, centrée sur le programme unificateur de l'analyse et d'un langage homogène, se répand dans toute l'Europe et devient une science respectée ${ }^{3}$. Pourtant, et bien qu'elle possède son propre " espace épistémologique " ${ }^{4}$, rares sont les études historiques sur l'enseignement de cette discipline dans le supérieur au XIX siècle $^{5}$. Un premier

1 René Taton (dir.), Enseignement et diffusion des sciences en France au XVIII ${ }^{2}$ siècle, Paris, Hermann, 1986. Voir aussi l'article de Christine Lehman dans ce numéro.

2 Bernadette Bensaude-Vincent, Isabelle Stengers, Histoire de la chimie, Paris, La Découverte, 1993.

3 Bernadette Bensaude-Vincent, Ferdinando Abbri (dir.), Lavoisier in European Context. Negotiating a New Language for Chemistry, Canton, Watson, 1995.

4 Roger Barlet, "L'espace épistémologique et didactique de la chimie ", Bulletin de l'union des physiciens, vol. 93, octobre 1999, n 817, p. 1423-1448.

5 André Grelon, "Pour une histoire des formations à la chimie en France ", in Georges Bram et al. (dir.), La chimie dans la société : son rôle, son image, actes du colloque interdisciplinaire du Comité national de la recherche scientifique, Biarritz, 23-25 mars 1994, Paris, L’Harmattan/CNRS, 1995, p. 21-32. 
travail de synthèse a été réalisé par François Leprieur ${ }^{6}$. Parmi les travaux plus récents, un ouvrage a été consacré aux manuels ${ }^{7}$, une étude à l'enseignement de la chimie à l'École polytechnique ${ }^{8}$, une autre à Louis-Jacques Thénard au Collège de France ${ }^{9}$ et une autre encore à l'École de pharmacie ${ }^{10}$. Pour la seconde moitié du XIX ${ }^{\mathrm{e}}$ siècle, a été étudiée l'action de Charles Adolphe Wurtz à la Faculté de médecine et d'Edme (dit Edmond) Frémy au Muséum national d'histoire naturelle ${ }^{11}$.

Comme dans tous ces travaux, on abordera ici la question de l'enseignement sous l'angle d'une histoire matérielle : cette approche mêle l'analyse du contenu des cours et l'étude de leur mise en œuvre effective ; elle s'intéresse aussi bien au matériel pédagogique proprement dit (manuels, instruments, accessoires de diverses natures comme les tableaux ou affiches) ou à la prise de notes qu'à l'organisation et à la gestion en objets et en personnels des chaires considérées.

Une caractéristique fondamentale de l'enseignement de la chimie réside dans la nature duale des cours héritée du siècle des Lumières. Depuis la publication des Élémens de chymie théorique du médecin chimiste Pierre Joseph Macquer en 1749, suivis des Élémens de chymie pratique en 1751, ces cours comportent une partie théorique, ou " philosophie chimique ",

6 François Leprieur, Les conditions de la constitution d'une discipline scientifique, la chimie organique en France, 1830-1880, thèse de $3^{\mathrm{e}}$ cycle, université Paris 1-Sorbonne, 1977 ; id., " La formation des chimistes français au XIX ${ }^{\mathrm{e}}$ siècle ", La Recherche, juillet-août 1979, vol. 10, n 102 , p. 732-740.

7 Bernadette Bensaude-Vincent, Antonio García Belmar, José Ramón Bertomeu-Sánchez, L'émergence d'une science des manuels. Les livres de chimie en France (1789-1852), Paris, Éditions des archives contemporaines, 2003.

8 Hélène Tron, "Chimie et laboratoires à l'École polytechnique du Palais-Bourbon (1794-1805) ", Sabix, $\mathrm{n}^{\circ}$ 15, juin 1996.

9 Antonio García Belmar, "The didactic Use of Experiment, Louis-Jacques Thenard's Lectures at the Collège de France ", in J. R. Bertomeu Sánchez, A. Nieto-Galán (dir.), Chemistry, Medecine and Crime. Mateu J. B. Orfila (1787-1853) and His Times, Sagamore Beach, Science History Publications, 2006, p. 25-53.

10 Sacha Tomic, " La gestion d'un laboratoire de chimie. L'exemple de l'École de pharmacie de Paris sous la direction de Vauquelin (1803-1829) ", journée d'étude sur les espaces de recherche et d'enseignement de la chimie à Paris aux XVIII ${ }^{\mathrm{e}}$ et XIX ${ }^{\mathrm{e}}$ siècles, 7 avril 2009. Sur le rôle crucial des pharmaciens dans l'histoire de la chimie, voir Jonathan Simon, Pharmacy, Chemistry and Revolution in France, 1777-1809, Aldershot, Ashgate, 2005 ; Sacha Tomic, Aux origines de la chimie organique. Méthodes et pratiques des pharmaciens et des chimistes, 1785-1835, Rennes, Presses universitaires de Rennes, 2010, Coll. "Carnot".

11 Ana Carneiro, The Research School of Chemistry of Adolphe Wurtz, Paris, 1853-1884, Ph. D., University of Kent, Canterbury, 1992 ; Natalie Pigeard, Ana Carneiro, "Chimistes alsaciens au XIX siècle, un réseau, une école? ", Annals of Science, vol. 54, 1997, p. 545 ; Danielle Fauque, "Organisation des laboratoires à Paris sous le ministère Duruy (1863-1869). Cas des laboratoires de Frémy et de Wurtz ", Annals of Science, vol. 62, n 4, octobre 2005, p. 501-531. 
et une partie pratique. Cette spécificité, partagée avec la physique, s'incarne dans le caractère bicéphale de l'enseignement de la chimie. Au XVIII ${ }^{\mathrm{e}}$ siècle ce sont les professeurs de la Faculté de médecine qui assurent la partie théorique du cours et les apothicaires qui, sous le titre de "démonstrateurs ", sont en charge de la "démonstration expérimentale " (préparation et exposition des expériences justifiant la théorie) ${ }^{12}$. Les cours magistraux se déroulent dans l'amphithéâtre et les expériences qui les accompagnent se préparent au laboratoire. Cette structure, commune à l'ensemble des établissements, constitue le socle de l'enseignement de la chimie et amène à considérer le laboratoire comme une extension naturelle de l'amphithéâtre. L'étude de l'organisation matérielle de ces deux espaces et de l'évolution de leurs relations est nécessaire pour saisir les modalités d'un cours magistral de chimie offrant un " parcours complet [de ce] domaine du savoir ", selon la définition proposée par Annie Bruter ${ }^{13}$.

À la fin du XVIII ${ }^{e}$ siècle deux méthodes d'enseignement sont en concurrence : celle qui suit l'ordre classique hérité de l'histoire naturelle et consiste à organiser les cours selon les trois règnes (minéral, végétal, animal); et la "méthode analytique " exposée par Antoine Laurent de Lavoisier dans son Traité élémentaire de chimie en 1789, qui présente la chimie selon une logique de complexité croissante, elle-même secondée par un " ordre des connaissances " qui va du connu à l'inconnu. De plus, Lavoisier entend accélérer l'apprentissage de la chimie en substituant une formation pratique où les élèves manipulent produits et instruments de mesure (thermomètre, balance, etc.) à la longue habitude par laquelle les apprentis chimistes acquéraient un " thermomètre au bout des doigts " et un " coup d'œil " d'artiste ${ }^{14}$. Pourtant, la méthode qu'il préconise ne s'est généralisée qu'à la fin du siècle suivant, laissant pour longtemps le cours magistral assurer seul l'enseignement de la chimie. Pourquoi ? Dans quelles conditions les professeurs du XIX ${ }^{e}$ siècle ont-ils mis en pratique les préceptes pédagogiques hérités des Lumières?

Pour répondre à ces questions, notre étude se fonde sur une dizaine d'établissements parisiens où elle est enseignée, soit en tant que telle, soit comme " science accessoire " servant aux sciences naturelles (minéralogie, géologie,

12 Voir l'article de Christine Lehman dans ce numéro.

13 Annie Bruter, "Le cours magistral comme objet d'histoire ", Histoire de l'Éducation, n 120, octobredécembre 2008, p. 11.

14 Bernadette Bensaude-Vincent, Isabelle Stengers, Histoire de la chimie, op. cit., p. 121. 
métallurgie), aux sciences médicales (matière médicale, pharmacie, médecine légale, toxicologie, hydrologie, hygiène, biologie) ou à l'industrie (chimie industrielle, agricole, tinctoriale) ${ }^{15}$. Il n'est donc pas étonnant de trouver parmi les professeurs, outre des chimistes, des médecins, des pharmaciens, des naturalistes et des industriels.

On s'intéressera dans un premier temps à l'organisation spatiale et à quelques outils pédagogiques utilisés dans les amphithéâtres pour augmenter l'efficacité des cours magistraux. On abordera ensuite les questions économiques liées à la gestion d'une chaire de chimie, en estimant le montant des crédits d'enseignement tout en confrontant ces données aux témoignages de divers acteurs de l'époque. Enfin, on se penchera sur la mise en place progressive de ce complément au cours magistral que constituent les travaux pratiques.

\section{I - L'amphithéâtre et ses accessoires}

Comment les professeurs du XIX ${ }^{\mathrm{e}}$ siècle en charge d'une chaire de chimie améliorent-ils l'articulation entre cours théorique et démonstration expérimentale héritée du XVIII siècle ? Deux aspects sont à considérer : l'adaptation de la configuration architecturale à cette articulation et la multiplication des outils pédagogiques, en particulier des supports visuels.

\section{1 - L'alliance de l'amphithéâtre et du laboratoire}

L'agencement des locaux n'est pas étranger aux facilités d'un enseignement expérimental de la chimie et à la présentation à l'auditoire d'expériences " esthétiques " et " démonstratives ". À l'École normale supérieure, Henri Sainte-Claire Deville dispose en 1868 "d'un vaste laboratoire d'enseignement, en communication directe avec les laboratoires consacrés à la préparation de démonstration

15 Muséum national d'histoire naturelle, École polytechnique, Conservatoire national des arts et métiers, Collège de France, École nationale vétérinaire d'Alfort, Faculté des sciences, Faculté de médecine, École des mines, École supérieure de pharmacie, École centrale des arts et manufactures, École normale supérieure, Institut national agronomique, École municipale de physique et de chimie industrielles de la ville de Paris, Institut de chimie appliquée. L'évolution des chaires de chimie correspondantes et de leurs poids relatifs dans l'enseignement supérieur fera l'objet d'un article ultérieur. 
pour les cours " ${ }^{16}$. Cette proximité entre l'espace réservé au cours et à celui de sa préparation n'est pas nouvelle. Elle existait déjà au Muséum national d'histoire naturelle, où le nouvel amphithéâtre construit par Edme Verniquet en 1787 était mitoyen avec " une vaste salle rectangulaire destinée à servir de laboratoire : on y plaça des fourneaux et tout le matériel nécessaire pour les cours. La chimie restait donc annexée à l'amphithéâtre " ${ }^{17}$.

C'est aussi le cas, à partir de 1801, au Collège de France, où opère le pharmacien-chimiste Nicolas Louis Vauquelin (1763-1829), puis Thénard à partir de $1804^{18}$. Dès son arrivée, Thénard agrandit l'espace d'enseignement en multipliant les dépendances de l'amphithéâtre. Il y présente pendant son cours des expériences minutieusement préparées dans l'" atelier de chimie " par son préparateur : elles arrivent après l'explication théorique, ne laissant place à aucune improvisation ${ }^{19}$, car cette pédagogie de la " démonstration expérimentale " exige leur réussite (d'après son fils, Thénard " ne faisait qu'un nombre restreint d'expériences bien choisies, mais il les voulait rigoureuses, frappantes, arrivant au moment précis ${ }^{20}$ ). Choisies en fonction de leur intérêt didactique (exposer, constater et expliquer les faits), elles servent à présenter les propriétés chimiques et physiques de manière "palpable ", et aussi à montrer les instruments, afin que l'élève puisse reproduire les expériences avec ses propres moyens (dans des laboratoires de fortune ou des cours privés). Thénard s'illustre également à l'École polytechnique où, selon l'élève Bosquet écrivant à sa mère en 1829, "il y a un amphithéâtre particulier pour la chimie, plus vaste que les autres, et dans lequel Thénard fait sous nos yeux ses expériences ${ }^{21}$.

16 Catherine Paquot, Henri Sainte-Claire Deville. Chimie, recherche et industrie, Paris, Vuibert/Institut pour l'histoire de l'aluminium, 2005, p. 133 (c'est moi qui souligne) ; J. Serre, "Le laboratoire de chimie de l'ENS de 1811 à 1954 ", Bulletin de la société des amis de l'École normale supérieure, n² 213, juillet 1999, p. 3-11.

17 Victor Plouvier, " Historique des chaires de Chimie, de Physique végétale et de Physiologie végétale du Muséum d'Histoire Naturelle ", Bulletin du Muséum d'histoire naturelle, vol. 3, 1981, p. 93-155 (c'est moi qui souligne).

18 José Ramon Bertomeu Sánchez, Antonio García Belmar, "Louis Jacques Thenard’s Chemistry Courses at the Collège de France, 1804-1835 ", Ambix, vol. 57, nº 1, mars 2010, p. 48-63 ; Abel Lefranc, Histoire du Collège de France depuis ses origines jusqu'à la fin du premier Empire, Paris, Hachette, 1893.

19 Antonio Garcia Belmar, "The didactic Uses of Experiment... ", art. cit., p. 25-53.

20 Paul Thénard, Un grand français, le chimiste Thénard, 1777-1857, Dijon, Jobard, 1950, p. 107.

21 Gaston Pinet, Histoire de l'École polytechnique, Paris, Librairie Polytechnique Baudry, 1887, p. 461. 
Ce dispositif s'étend à tous les lieux d'enseignement de la chimie au cours du siècle. Nommé professeur à l'École nationale vétérinaire d'Alfort en 1813, le physicien et chimiste Pierre-Louis Dulong divise le laboratoire de pharmacie afin d'y installer un laboratoire de chimie en communication avec un nouvel amphithéâtre $^{22}$. À la faculté de médecine, un auditeur anglais, Joseph Ince, décrivant le cours de chimie médicale du médecin chimiste Mathieu Orfila, est frappé " par la quantité considérable d'expériences dont les préparatifs encombraient la table et qui se succédaient, pour ainsi dire, sans fin ${ }^{23}$. Lors de l'agrandissement de l'École supérieure de pharmacie en 1839, on abandonne l'ancienne structure, qui confondait laboratoire et amphithéâtre, pour une nouvelle disposition qui les distingue (ill. 1) ${ }^{24}$. Celle-ci sera conservée lors de la reconstruction de l'école sur son nouveau site en 1882 .

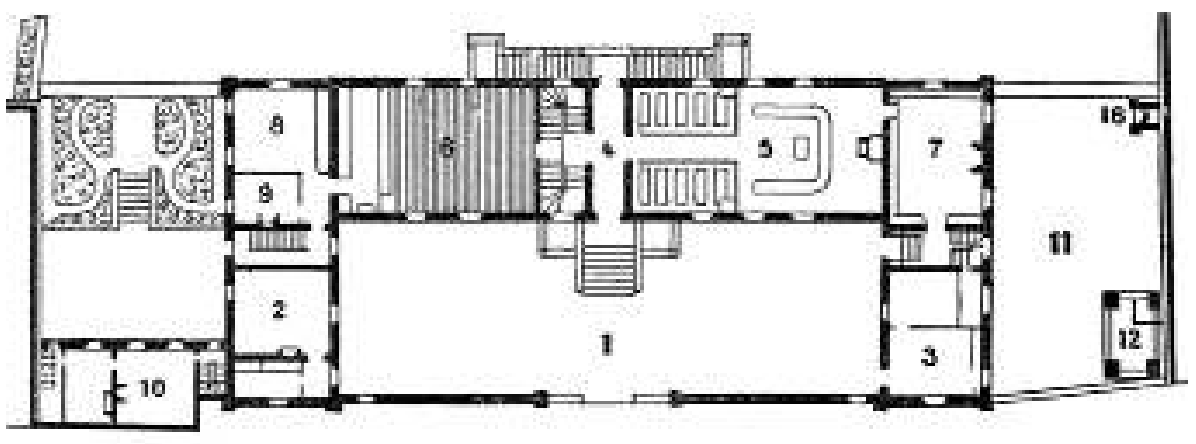

Ill. 1 : plan de l'école de pharmacie en 1839

(Centenaire de l'École supérieure de pharmacie de l'Université de Paris, 1803-1903, Paris, A. Joanin et Cie, 1904)

L’École des mines, reconstruite en 1866, bénéficie également, pour l'année préparatoire, d'une salle adossée à l'amphithéâtre pour la préparation des cours de chimie $^{25}$. L'évolution est identique à l'École centrale des arts et manufactures. Entre l'ancienne situation, où les deux espaces étaient séparés, et la mise en œuvre des nouveaux plans, il a été établi en 1885 " un laboratoire pour la pré-

22 Alcide Raillet, Léon Moulé, Histoire de l'École d'Alfort, Paris, Asselin et Houzeau, 1908, p. 763.

23 Joseph Ince, "Le cours d'Orfila ", Journal de chimie médicale, de pharmacie et de toxicologie, t. 9, 1853, p. 789.

24 L'amphithéâtre (6) communique avec le laboratoire (8) et le cabinet du professeur (9).

25 Adolphe Carnot, "Les laboratoires de l'École nationale des mines ", Journal de chimie médicale, de pharmacie et de toxicologie, t. IX, 1853, p. 792. 
paration des cours de chimie à proximité de l'amphithéâtre correspondant, ce qui facilite beaucoup la mise en train des expériences en les rendant d'ailleurs plus sûres et plus rapides ${ }^{26}$. L'amphithéâtre, qui peut recevoir 250 élèves, dispose d'un éclairage " à la lumière Edison ", de trois grands " tableaux ardoisés " que l'on peut déplacer grâce à un système hydraulique et d'une "large table d'expériences avec l'eau, le gaz, la vapeur, l'électricité ; d'autres tuyaux amènent divers gaz, le vide et l'air comprimé " (ill. 2) ${ }^{27}$.

À la faculté des sciences, cette disposition des lieux n'est mise en place que très tardivement. Dès son rapport de 1837, le futur doyen JeanBaptiste Dumas (1800-1884) déclarait qu'il faudrait "à la Faculté des sciences un amphithéâtre de chimie [séparé de celui de physique] susceptible d'être mis en communication avec le laboratoire, de telle sorte que toutes les opérations pussent être présentées aux élèves au moment opportun et sur une grande échelle "28. Ce vœu ne sera exaucé que soixante ans plus tard, lors de l'installation de la faculté des sciences en 1895 dans la Nouvelle Sorbonne, œuvre de l'architecte Henri-Paul Nénot (qui travaille en étroite collaboration avec les professeurs). Ce dernier décrit le laboratoire de préparation des cours, qui contient, en son milieu, " une table carrelée sur laquelle il est possible de monter les grands appareils qui doivent figurer aux leçons " ${ }^{29}$. Cette salle est centrale car elle est non seulement attenante à l'amphithéâtre, dont " la pente des gradins est très accentuée pour laisser voir facilement les expériences qui se font sur la table de cours ", mais également en communication directe avec les trois autres services de chimie (générale, minérale et organique) ${ }^{30}$.

26 Charles de Comberousse, Histoire de l'École centrale des arts et manufactures depuis sa fondation jusqu'à nos jours, Paris, Gauthier-Villars, 1879, p. 301.

27 Émile Bouant, Dictionnaire de chimie, Paris, Librairie J.-B. Baillière, 1888, p. 539.

28 Jean-Baptiste Dumas, "Rapport adressé au Ministre de l'Instruction Publique au nom de la Faculté des sciences ", in Octave Gréard, Éducation et instruction. Enseignement supérieur, Paris, Hachette, 1889, p. 246.

29 Henri-Paul Nénot, La Nouvelle Sorbonne, Paris, A. Colin, 1895, p. 39.

30 Ibid., p. 54. 

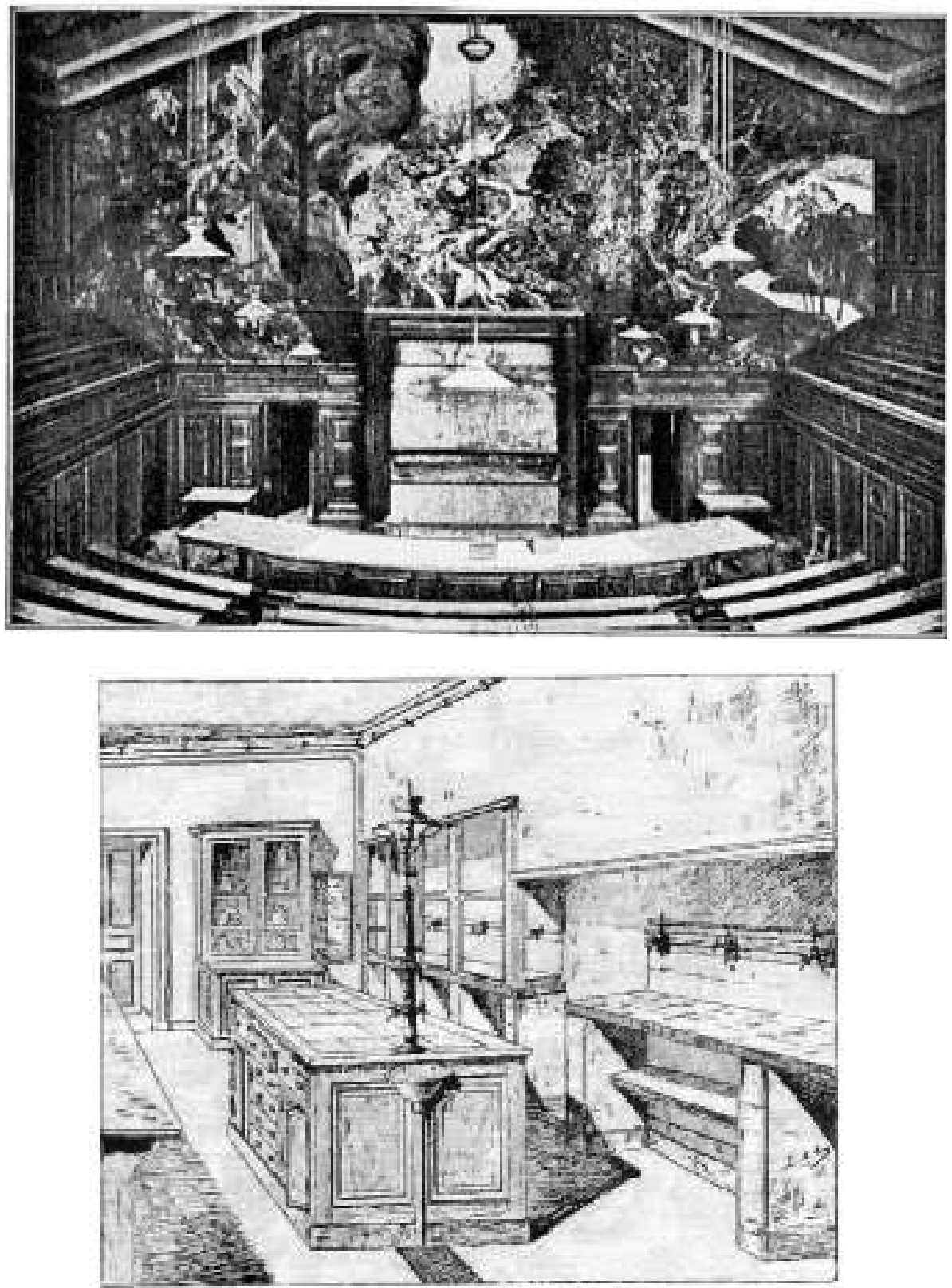

Ill. 2 : amphithéâtre de chimie et laboratoire de la préparation des cours de la Faculté des sciences

(En haut : Maurice Caullery, «La Faculté des sciences » in La vie universitaire à Paris, Paris, A. Colin, 1918 ; en bas : H.-P. Nénot, La Nouvelle Sorbonne, Paris, A. Colin, 1895, p. 39) 
Cette disposition en deux espaces pédagogiquement et physiquement inséparables sera le modèle architectural le plus répandu au XIX ${ }^{\mathrm{e}}$ siècle dans de nombreux pays européens : par exemple en Allemagne, à l'Université de Berlin ou à l'Institut de chimie de Graz, mais également en Hongrie, à Budapest ${ }^{31}$.

\section{2 - Des supports visuels variés}

Si l'association de l'amphithéâtre et du laboratoire facilite sans conteste la présentation et la fréquence des "démonstrations " d'expériences, les professeurs vont augmenter l'efficacité des cours magistraux grâce à divers supports visuels.

L'une des premières, la table des affinités établie en 1718 par l'apothicairechimiste Étienne François Geoffroy (1672-1731) ${ }^{32}$, est expliquée dans les cours et sert de support pédagogique. Au XIX ${ }^{\mathrm{e}}$ siècle, la mise en tableau du savoir chimique s'enrichit de divers graphiques et affiches. Selon le témoignage du chimiste et physicien anglais Michael Faraday, en visite à Paris en 1813, les leçons de Louis Joseph Gay-Lussac à l'École polytechnique sont agrémentées de "graphiques sommaires et d'expériences "33. Il y a aussi des tableaux à la faculté des sciences où, faute de matériel, le chimiste privilégie un enseignement abstrait ${ }^{34}$. Au Collège de France, " l'immense tableau noir [est] surmonté lui-même de trois autres tableaux où sont inscrits les noms des métaux, des métalloïdes et des acides avec leurs symboles et leurs équivalents " ${ }^{35}$. En 1843, une notice de l'ingénieur chimiste et répétiteur de chimie à l'École centrale des arts et manufactures, David Clovis Knab, précise que "sur la demande de professeurs célèbres, des Tableaux d'une grande dimension [ $1 \mathrm{~m} 70$ sur $1 \mathrm{~m} 45]$ servent maintenant à Paris aux Cours de Chimie et de Physique de la Sorbonne, du Collège de France, de l'École Polytechnique, et du Conservatoire royal des Arts et Métiers ${ }^{36}$. Dans un rapport présenté

31 Georges Quesneville, "Le laboratoire de chimie de l'Université Frédéric-Guillaume à Berlin ", Moniteur scientifique, 252 livraison, 15 juin 1867, p. 497-516 ; Adolphe Wurtz, Les hautes études pratiques dans les universités d'Allemagne et d'Autriche-Hongrie, Paris, G. Masson, 1882, p. 58-59 ; Gaston Bonnier, "Les laboratoires scientifiques à l'étranger ", La Nature, n 366, 5 juin 1880, p. 2-6.

32 Voir la reproduction de ce tableau dans l'article de Christine Lehman.

33 Maurice Crosland, Gay-Lussac 1778-1850, savant et bourgeois, Paris, Belin, 1991, p. 216.

34 Ibid., p. 214.

35 Alexis Lemaistre, L'Institut de France et nos grands établissements scientifiques : Collège de France, Muséum, Institut Pasteur, Sorbonne, Observatoire..., Paris, Hachette, 1896, p. 103.

36 David Clovis Knab, "Tableaux peints destinés à l'enseignement " in Jean-Baptiste Dumas, Traité de chimie appliquée aux arts, Paris, Béchet, t. 6, 1843, p. 1. 
par l'ingénieur Amédée Durand à la Société pour l'encouragement de l'industrie nationale, l'initiative de Knab est jugée " d'une haute portée par l'efficacité de son action sur l'enseignement ${ }^{37}$, en particulier en chimie industrielle. $\mathrm{Au}$ Conservatoire national des arts et métiers, le "manufacturier-chimiste " Anselme Payen fait usage des tableaux et en assure la promotion auprès de ses collègues, Knab se chargeant de la description des appareils de chimie appliquée et de la légende des lithographies du cours ${ }^{38}$. Thénard, de son côté, insiste sur " un point fort essentiel ", celui de donner à chaque élément représenté sur l'image " la couleur naturelle qui lui est propre : le dessin, par cela même, devient bien plus intelligible $" 39$. Guidé par le même souci de réalisme que ses collègues, le professeur de chimie de l'École nationale vétérinaire d'Alfort, Jean-Louis Lassaigne, est le premier à publier des " tableaux synoptiques où sont figurés, avec leur couleur naturelle, les précipités formés par les réactifs. [...] Ils retraceront toujours aux yeux les teintes si variables et si difficiles à décrire. [...] Ils représenteront à tous moments aux élèves les effets dont ils auront été les témoins dans les cours qu'ils ont suivis "40. L'usage de couleurs est particulièrement apprécié pour l'écriture des formules développées qui se multiplient en fin de siècle. À l'École supérieure de pharmacie, les garçons de laboratoire, voire certains élèves, "étaient chargés de préparer les tableaux que le maître allait utiliser à l'amphithéâtre : ils traçaient, avec des craies de couleurs, les formules chimiques nécessaires à la leçon " ${ }^{41}$.

À la fin du siècle, de nombreux établissements bénéficient des progrès de la photographie et des appareils de projection, qui répondent au souci de réalisme tant recherché par les professeurs. Selon les membres du conseil d'administration de la Société pour l'encouragement de l'industrie nationale, "la lanterne magique est connue depuis très longtemps. Dans ces derniers temps on a varié ses effets et on s'en est beaucoup servi aux cours publics

37 A. Durand, "Rapport au nom de la commission du Bulletin, sur des figures ou dessins destinés aux démonstrations dans l'enseignement des sciences, présenté par M. Knab, rue de Vendôme, 11 ter ", Bulletin de la Société d'encouragement pour l'industrie nationale, t. 42, 1843, p. 21.

38 Bulletin de la Société d'encouragement pour l'industrie nationale, t. 41, 1842, p. 466 ; Anselme Payen, Cours de chimie organique appliquée. Description des appareils de chimie appliquée, légende des lithographies du cours, par MM. Knab... et Leblanc..., Paris, Conservatoire national des Arts et métiers, 1842.

39 Ibid., p. 3 ; Louis-Jacques Thenard, Extrait du rapport lu à l'Académie des sciences.

40 Jean-Louis Lassaigne, Abrégé élémentaire de chimie, considérée comme science accessoire à l'étude de la médecine, de la pharmacie et de l'histoire naturelle, Paris, Béchet, 1829, p. X.

41 Floriane Blanc, Philippe Jaussaud, "Les leçons inaugurales de chimie des pharmaciens français ", Revue d'histoire de la pharmacie, t. 55, n 353, 2007, n. 21. 
de la Sorbonne " ${ }^{42}$. Tel est également le cas à l'Institut national agronomique ${ }^{43}$, dans les nouveaux amphithéâtres de l'École supérieure de pharmacie ou à l'École centrale des arts et manufactures, où " les tableaux peuvent se relever complètement et découvrir une glace dépolie, sur laquelle on fait des projections par transparence, l'appareil projecteur étant placé dans un cabinet attenant à l'amphithéâtre " ${ }^{44}$.

Un autre outil pédagogique agite le monde des chimistes : les représentations atomiques, ancêtres de nos modèles moléculaires. Réhabilitée dans les années 1800 par l'anglais John Dalton (1766-1844), la notion d'atome fut d'abord acceptée, puis rejetée dans les années 1830 par les chimistes français, Jean-Baptiste Dumas en tête. Les Anglais et les Allemands privilégiant la théorie atomique, des divergences apparurent, en particulier dans les notations des composés chimiques. Ces confusions furent à l'origine du premier congrès international de chimie, qui se tint à Karlsruhe en 1860. Un certain nombre de chimistes se convertirent à l'atomisme mais il n'y eut pas de consensus $^{45}$. Le développement de la chimie structurale dans les années 1860 et les polémiques autour de la théorie atomique ont contribué à donner aux étudiants français une image brouillée de la chimie puisque s'opposaient, d'un côté, l'école des anti-atomistes, ou " équivalentistes ", menés par le pharmacienchimiste Marcelin Berthelot, ${ }^{46}$, de l'autre, celle des atomistes sous la houlette de Wurz ${ }^{47}$. Entre 1860 et 1884, eurent lieu de nombreuses controverses restées célèbres. Après s'être réfugié derrière un "positivisme polémique " ${ }^{48}$, Berthelot finira par se convertir définitivement à l'atomisme en avril $1896^{49}$.

42 Séance du 23 avril 1869, Bulletin de la Société d'encouragement pour l'industrie nationale, t. 16, 1869, p. 511.

43 Léon Dabat, Rapport du jury international. Groupe I - Éducation et enseignement. Classe 5 : enseignement spécial agricole, Paris, Imprimerie nationale, 1904, p. 120-121.

44 Émile Bouant, Dictionnaire de chimie, op. cit., p. 539.

45 Bernadette Bensaude-Vincent, "Karlsruhe, septembre 1860 : l'atome en congrès ", Relations internationales, $n^{\circ}$ 62, été 1990, p. 153-160.

46 En faisaient notamment partie Deville à l'École normale supérieure, Frémy au Muséum national d'histoire naturelle et Georges Lemoine à l'École polytechnique.

47 Wurz convertit Édouard Grimaux, qui enseigna la théorie atomique pour la première fois à l'École polytechnique en 1881-1982.

48 Bernadette Bensaude-Vincent, "Atomism and Positivism: A Legend About French Chemistry ", Annals of Science, vol. 56, 1999, p. 90.

49 Alan J. Rocke, Nationalizing Science. Adolphe Wurtz and the Battle for French Chemistry, Cambridge, Massachusetts Institute of Technology Press, 2000, p. 281 et 330. 
Pour convaincre son auditoire, Wurtz utilise pour la première fois, lors du congrès de l'Association française pour l'avancement des sciences, à Clermont en 1876, des petites boules formant un modèle atomique (ce type de modèle a été introduit au cours des années 1860 par les chimistes anglais et allemands, en particulier par August Hofmann) ${ }^{50}$. Contrairement aux affiches et projections, il s'agit, cette fois, de la représentation d'une notion abstraite, celle des atomes, suggérant une conception naïvement réaliste de ceux-ci qui est loin de faire l'unanimité chez les chimistes français, y compris parmi les atomistes. Bien qu'il soit difficile de connaitre la fréquence d'utilisation de ces modèles moléculaires primitifs durant les leçons, c'est bien au nom de l'efficacité pédagogique que de tels outils ont été introduits, en particulier pour la compréhension de la notion de valence et la synthèse des composés organiques. De même qu'il a entraîné la construction de locaux spécifiques, le développement du cours magistral de chimie au XIX ${ }^{\mathrm{e}}$ siècle a suscité l'utilisation des ressources visuelles diverses que le progrès technique et pédagogique mettait à la disposition des professeurs.

\section{II - Grandeur et misère des budgets}

Comme au siècle des Lumières, la préparation d'un cours comportant des expériences nécessite une logistique qui implique plusieurs acteurs transitant entre l'amphithéâtre et le laboratoire. Cette organisation va se complexifier au cours du siècle.

\section{1 - Des chaires coûteuses en personnel et en matériel}

Derrière le professeur il y a toute une équipe à ses ordres. Le professeur est assisté ou remplacé dans son cours magistral par son "assistant " ou " adjoint ", comme à l'École supérieure de pharmacie, ou bien par un " maître de conférences ", comme à l'École normale supérieure à partir de 1815 et à la faculté des sciences après $1877^{51}$. Des agrégés, nommés sur concours pour une période déterminée (généralement cinq à dix ans), viennent compléter

50 Natalie Pigeard, Ana Carneiro, "Chimistes alsaciens au XIXe siècle : un réseau, une école? ", art. cit., p. 545 ; Christophe Meinel, "Molecules and Croquet Balls " in Soraya de Chadarevian, Nick Hopwood (dir.), Models. The Third Dimension of Science, Stanford, Stanford University Press, 2004, p. $242-275$.

51 Les maitres de conférences sont supprimés à l'ENS lors de son intégration à l'Université de Paris en 1903 et les adjoints à l'École de pharmacie en 1874. 
les enseignements, ainsi à la faculté de médecine à partir de 1829 ou à l'École supérieure de pharmacie et à la faculté des sciences après $1840^{52}$.

Quant à l'organisation du laboratoire, elle obéit à une hiérarchie qui comporte de trois à quatre niveaux selon l'établissement. À l'École polytechnique, l'instituteur-professeur dirige le " conservateur du magasin " ou " préparateur général ", qui est secondé dans son travail par des " artistes chimistes " ou " préparateurs particuliers ", eux-mêmes suppléés par des " aides de laboratoire ". Ailleurs, le professeur, qui assure parfois la fonction de directeur du laboratoire, a sous ses ordres directs les " chefs des travaux " ou "sous-chefs ", également appelés " aides-naturalistes " ou " aides-chimistes ", comme au Muséum national d'histoire naturelle depuis 1802, puis " assistants " à partir de 1891, ou encore " agrégés préparateurs " à l'École normale supérieure depuis leur institution en 1847. Ces derniers dirigent les " aides préparateurs " et autres " préparateurs des cours adjoints " ou " particuliers ". Enfin, en bout de chaîne, les "garçons " de laboratoire assistent les préparateurs.

Le nombre des personnels ne cessera d'augmenter tout au long du siècle. À titre d'exemple, aux débuts de l'École supérieure de pharmacie, il n'y avait qu'un préparateur (et un garçon) pour trois cours (chimie, pharmacie, matière médicale). Leur nombre passe à deux en 1839, à trois en 1858, à quatre en 1868 - date à laquelle apparait le titre de " chef des travaux chimiques et pharmaceutiques " - et il explose en 1876, passant à douze. Toutes ces personnes sont rétribuées et leurs tâches et compétences sont précisées par la loi ou lors des assemblées des professeurs. Cette organisation amplifie singulièrement le duo professeur/démonstrateur en œuvre au XVIII siècle. Il y a donc, autour d'une chaire d'enseignement de la chimie, un véritable enjeu financier qu'il convient de préciser.

La misère du budget de l'Instruction publique pour l'enseignement supérieur est bien connue des historiens. La création de l'École pratique des hautes études (EPHE) à la fin du Second Empire amorce un premier engagement timide de l'État, que la III ${ }^{e}$ République va consolider grâce un investissement massif, mettant ainsi fin à la "longue stagnation des années 1800-1880 "53. À partir de données dispersées dans différents rapports et ouvrages com-

52 Georges Dillemann, "Professeurs agrégés de l'École supérieure de pharmacie de Paris ", Revue d'histoire de la pharmacie, t. 34, $n^{\circ} 274$, septembre 1987, p. 201-211. Sauf indication contraire, le mot " agrégé " désigne les agrégés du supérieur, que l'on ne doit pas confondre avec ceux du secondaire dont le premier concours est organisé en 1821.

Antoine Prost, Histoire de l'enseignement en France, 1800-1967, Paris, A. Colin, 1968, p. 223-229. 
mémoratifs ${ }^{54}$, il apparaît que deux grandes catégories d'établissements peuvent être distinguées : dans la première, ceux dont le budget passe le cap du demi-million après 1850 (l'École polytechnique, la faculté de médecine, le Muséum, la faculté des sciences, l'École centrale) ; dans la seconde, ceux dont le budget converge autour du demi-million de francs en fin de siècle (l'ENS, l'École nationale vétérinaire d'Alfort, le Collège de France, l'Institut national agronomique, le Conservatoire national des arts et métiers, l'École supérieure de pharmacie), l'École des mines bénéficiant des crédits les plus faibles (250 000 francs en 1900) ${ }^{55}$.

Sur ce budget global, le traitement du personnel constitue généralement le premier poste de dépenses. Celui-ci présente au début du siècle des disparités qui vont progressivement s'atténuer et pratiquement disparaitre à partir des années 1870. Jusqu'en 1877, les professeurs perçoivent une part de traitement fixe, augmentée d'une part "d'éventuel " plus ou moins importante qui dépend en grande partie des frais d'examen et de réception des candidats : c'est le cas à la faculté de médecine, à l'École supérieure de pharmacie ou à la faculté des sciences, véritable " machine à examens " selon l'expression de Louis Liard ${ }^{56}$. Selon les établissements, les traitements vont de 1500 à 3000 francs vers 1800, de 4000 à 6000 francs au milieu du siècle et atteignent de 10000 à 15000 francs à partir des années 1870. Les adjoints prennent également part aux examens et perçoivent une indemnité de 1000 à 3000 francs, somme pratiquement équivalente pour les chefs des travaux et certains préparateurs, ce qui témoigne de leur importance au sein du dispositif. Enfin, les garçons ont des gages de l'ordre de 500 à 1000 francs. On peut donc estimer que le budget minimal en personnel d'une chaire de chimie (un professeur, un adjoint, un directeur de laboratoire ou un chef des travaux, un préparateur et un aide de laboratoire) va d'environ 5000 à 15000 francs durant la plus grande partie du siècle, chiffres qui peuvent être grosso modo multipliés par trois à partir de 1870 .

54 Charles Jourdain, Le budget de l'instruction publique des établissements scientifiques et littéraires, Paris, Hachette, 1857 ; François Leprieur, Les conditions de la constitution d'une discipline scientifque..., op. cit., p. 3-42, ainsi que les comptes rendus rédigés lors des Expositions internationales et les ouvrages relatifs à l'histoire particulière d'une institution mentionnés dans cet article.

55 Charles Jourdain, Le budget de l'instruction publique..., op. cit., et François Leprieur, Les conditions de la constitution d'une discipline scientifique..., op. cit., p. 3-42.

56 Louis Liard, L'enseignement supérieur en France, 1789-1893, Paris, A. Colin, t. 2, 1894, p. 211. Le cumul des postes est un autre moyen qui permet d'augmenter substantiellement les revenus. Cette pratique est cependant marginale si on considère l'ensemble des professeurs titulaires. 
Quelle part du budget est consacrée au matériel ? Les frais d'installation permettant d'équiper un laboratoire oscillent de 3000 à 6000 francs vers 1800 (10 000 francs sont dépensés pour la rénovation du laboratoire du Collège de France en 1809) et se stabilisent à environ 10 000-15 000 francs à partir du milieu du siècle. Les frais pour l'entretien des équipements (verrerie et produits chimiques) varient de 1500 à 10000 francs par an, mais le plus souvent ils se situent entre 2500 et 5000 francs et doublent en fin de siècle. Une comparaison par établissements indique que les crédits alloués à l'enseignement de la chimie ne sont proportionnels ni au budget global ni à la taille d'un établissement. Le coût moyen annuel par élève peut être estimé à quelques dizaines de francs. Après la Révolution de 1848, certaines chaires disposent d'une centaine de francs, comme à l'École normale supérieure, particulièrement bien dotée grâce à la "libéralité de l'Empereur ", au Muséum national d'histoire naturelle ou à la faculté de médecine à partir des années 1860, ou bien encore à l'Institut de chimie appliquée en 1896, mais la plupart des établissements se contentent toujours de quelques dizaines de francs.

Finalement, le budget minimal d'une chaire de chimie, personnel inclus et hors installation, s'établit ainsi à 7 500-20 000 francs annuels jusqu'aux années 1870 et passe à 20 000-55 000 francs à l'orée du XXe siècle. Avant même de s'accompagner de travaux pratiques, l'enseignement magistral de la chimie est un enseignement coûteux.

\section{2 - Gérer la pénurie des moyens}

Les ordres de grandeur qui viennent d'être établis ne sauraient cacher la misère des équipements. En 1867, le futur inspecteur général Charles Jourdain (1817-1886) tire le signal d'alarme ${ }^{57}$. Si l'image du physiologiste Claude Bernard (1813-1878) décrivant les laboratoires comme les " tombeaux des scientifiques " a marqué les esprits, les chimistes ont également pesé de tout leur poids pour dénoncer leurs conditions de travail. Les diverses missions à l'étranger, en particulier celles de Wurtz en Allemagne et dans l'Empire austro-hongrois, ont permis d'alerter les autorités sur le décalage avec les puissants voisins de la France. Les laboratoires d'August Kékulé à Berlin, d'Hofmann dans la même ville et d'Hermann Kolbe à Leipzig sont cités

57 Charles Jourdain, Rapport sur l'organisation et les progrès de l'instruction publique, Paris, Imprimerie impériale, 1867, p. 208-209. 
en exemple ${ }^{58}$. Les sommes investies (plusieurs centaines de milliers de francs) donnent le vertige aux chimistes français. Ironie de l'histoire, c'est le modèle français du début du siècle qui avait inspiré le jeune Justus Liebig, lors de son séjour parisien au début des années 1820, pour la construction de sa célèbre école à Giessen. Cette école-prototype servira de modèle aux autres universités allemandes, et son organisation s'exportera outre-Atlantique, à Londres et bien sûr en France ${ }^{59}$. C'est ce que veut dire le directeur de la Revue scientifique Émile Alglave quand il déclare que l'Allemagne a "dérobé à la France le sceptre que celle-ci possédait encore sans conteste en 1830 " $^{60}$.

Ce jugement rétrospectif de la situation au début du siècle, présentée comme idyllique, concerne quelques pôles d'excellence comme l'École polytechnique, l'École supérieure de pharmacie (qui a hérité du matériel de l'ancien Collège de pharmacie) ${ }^{61}$, la faculté de médecine et le Collège de France. Seul point noir à ce tableau, la faculté des sciences, où Gay-Lussac est obligé d'apporter son propre matériel ${ }^{62}$. Cela explique l'orientation vers la "philosophie chimique " de ses cours, où les étudiants suivaient " avec peine les savantes leçons du professeur "63.

À la fin des années 1830, les conditions d'enseignement se dégradent. À l'École polytechnique, la chimie n'est guère valorisée, car réticente à une mathématisation poussée. Malgré la construction d'un nouveau laboratoire en 1840, son prestige décline et son enseignement sera même en " crise " au début du XX ${ }^{\mathrm{e}}$ siècle ${ }^{64}$. Victime de son succès, l'École supérieure de pharmacie a de plus en plus de mal à absorber le flux croissant d'élèves malgré divers agrandissements. À la faculté des sciences, la pénurie se poursuit et Dumas hérite d'un laboratoire " tout à fait insuffisant ". La création en 1855 du " laboratoire

58 José Freire, "Le laboratoire de l'Université de Leipzig ", La Nature, n² 219, 11 août 1877, p. 168-170.

59 William H. Brock, "Les nouvelles cathédrales de la science ", Les Cahiers de Science \& Vie, $\mathrm{n}^{\circ}$ 51, juin 1999, p. 26-33.

60 Émile Alglave, Revue des cours scientifiques de la France et de l'étranger, t. 6, 30 octobre 1869, p. 753.

61 Christian Warolin, "La création de l'École de pharmacie de Paris en 1803 ", Revue d'histoire de la pharmacie, t. 51, $\mathrm{n}^{\circ} 339,2003$, p. 465.

62 Maurice Crosland, Gay-Lussac..., op. cit., p. 225.

63 Auguste-Pierre Dubrunfaut, Revue encyclopédique, t. 47, 1830, p. 175.

64 Catherine Kounelis, "Heurs et malheurs de la chimie. La réforme des années 1880 ", in Bruno Belhoste, Amy Dahan Dalmenico et Antoine Picon (dir.), La formation polytechnicienne, 1794-1994, Paris, Dunod, 1994, p. 245-264 ; Micheline Charpentier-Morize, L. Nekoval-Chikkaoui, "Un enseignement en crise, la chimie dans la première moitié du XXe siècle ", ibid., p. 357-374. 
de perfectionnement et de recherches pour les études chimiques " s'avère vite inefficace, comme le note le doyen et zoologiste Henri Milne-Edwards ${ }^{65}$.

Les savants profitent de la défaite de 1870 pour dénoncer systématiquement la misère des équipements qui est, à leurs yeux, en grande partie responsable du désastre. Le chef des travaux chimiques au Collège de France, Alexandre-Joseph Riban, écrit en 1871 : « le Collège de France n'a pas un seul fourneau, donc il faudrait des fonds pour le construire. Nous avons un alambic mais pas de maçonnerie pour le supporter. Comment s'en servir ? "66. Il semble que le successeur à la chaire du pharmacien-chimiste Antoine Jérôme Balard, Paul Schützenberger, n'ait pas eu une audience très forte, du moins à la fin de sa carrière. Selon un témoin de l'époque, la salle de chimie, pourtant " bien installée ", servait " rarement " (figure 3$)^{67}$. On est loin des années fastes du professorat de Thénard, qui captivait plus de deux cents élèves, ou bien des leçons d'Orfila devant plus de mille auditeurs dans le grand amphithéâtre de la faculté de médecine. Dans les années 1870, Wurtz et son préparateur Georges Salet transportent leur propre matériel depuis la faculté de médecine pour pallier l'état pitoyable des moyens de la faculté des sciences, où enseigne Louis Troost et où Wurtz donne des leçons complémentaires de chimie organique ${ }^{68}$. En 1873, le rédacteur en chef de La Nature, Gaston Tissandier, critique dans le premier numéro de la revue le budget dérisoire consacré à l'entretien des facultés car "si l'argent est le nerf de la guerre, il est aussi celui de la science "69. Dix ans plus tard, Berthelot dénonce à son tour " les instruments vieillis et les laboratoires mesquins ou surannés " qui se trouvent dans des "baraquements " inégalement équipés ${ }^{70}$.

65 François Leprieur, Les conditions de la constitution d'une discipline scientifique..., op. cit., p. 41.

66 Ibid., p. 160.

67 On voit le professeur réalisant une expérience devant un public clairsemé, mais attentif, à l'image de l'auditeur au premier rang qui prend des notes.

68 Alan J. Rocke, Nationalizing Science. op. cit., p. 365 ; Octave Gréard, L'enseignement supérieur à Paris en 1881, Paris, Delalain, 1882, p. 34.

69 Gaston Tissandier, "L’enseignement supérieur ", La Nature, n 1, 1873, p. 2.

70 Marcelin Berthelot, "L'enseignement supérieur et son outillage ", Revue internationale de l'enseignement, t. 5, 1883, p. 383, n. 1 ; "Rapport sur les conférences faites à la Faculté des sciences de Paris ", Revue internationale de l'enseignement, t. 5, 1883, p. 322. 


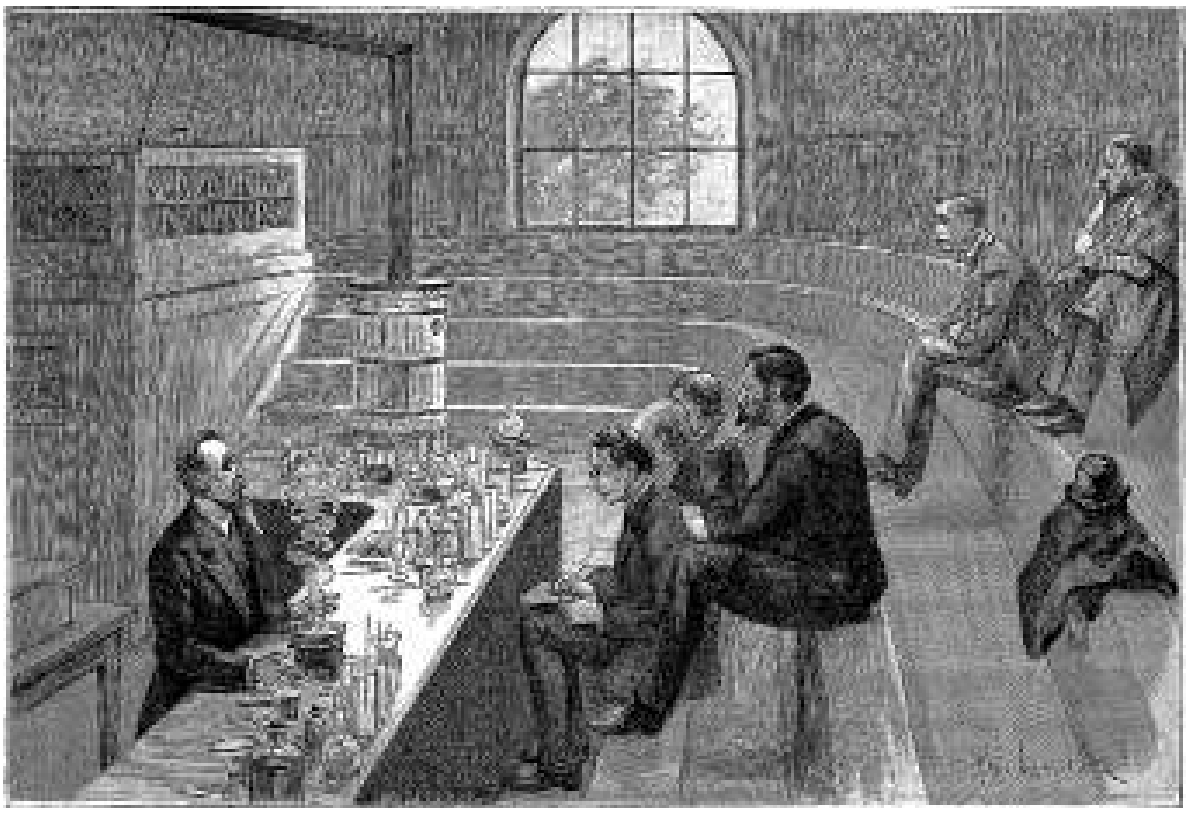

Ill. 3 : Paul Schützenberger lors d'un cours de chimie minérale au Collège de France.

(A. Lemaistre, L'Institut de France et nos grands établissements scientifiques, Paris : Librairie Hachette, 1896)

Mais la rivalité avec l'Allemagne consécutive à la défaite de 1871 pousse les responsables politiques à engager l'enseignement supérieur français dans la voie d'une modernisation qui permette à la France d'égaler son puissant voisin. La III ${ }^{e}$ République vient ainsi au secours des chimistes et investit des dizaines de millions de francs dans la rénovation et la reconstruction des établissements. Dans les années 1870, les laboratoires de Wurtz à la faculté de médecine et de Frémy au Muséum sont les premiers à disposer d'un équipement digne des standards allemands. La qualité de l'installation des nouveaux laboratoires du Muséum est telle que Gaston Tissandier (1843-1899) pourra écrire " qu'en deux ans la république aura fait plus pour le Muséum que l'empire pendant les vingt ans qu'il a présidé à nos destinées " ${ }^{71}$. C'est seulement à partir des années 1880, après la reconstruction de divers établissements, dont l'École supérieure de pharmacie en 1882, l'École centrale en 1885, l'École nationale vétérinaire d'Alfort en 1889, l'Institut national agronomique en 1890

71 Gaston Tissandier, "Les nouveaux laboratoires du Muséum d’histoire naturelle ", La Nature, $\mathrm{n}^{\circ} 1$, 1873 , p. 7. 
et la Nouvelle Sorbonne en 1895, que la chimie peut prétendre à des conditions d'enseignement décentes pour le cours magistral - et à la généralisation des travaux pratiques.

\section{III - L'articulation théorie-pratique : une pédagogie standard}

L'articulation théorie-pratique, c'est-à-dire la mise en place de travaux pratiques en complément des cours magistraux, qui est au cœur de la méthode d'enseignement préconisée par Lavoisier, est immédiatement appliquée à l'École polytechnique $^{72}$. Selon Thénard, alors répétiteur, "il est parfaitement démontré qu'il est impossible d'acquérir des connaissances précises en chimie sans faire soi-même des expériences chimiques ${ }^{73}$. Les élèves effectuent les montages et réalisent les expériences pour produire et étudier les substances abordées dans le cours. La construction d'un nouvel amphithéâtre en 1840 et l'extension du cabinet de chimie sont guidées par cette organisation pédagogique, car il s'agit de "laisser une trace durable dans la mémoire ", que l'enchaînement rapide des expériences du cours magistral ne peut assurer ${ }^{74}$. Malgré la place de plus en plus marginale accordée à la chimie, l'École polytechnique sera l'un des seuls établissements à maintenir des séances de travaux pratiques tout au long du siècle. C'est également le cas à l'École centrale des arts et manufactures, cette "Sorbonne industrielle " dont l'organisation s'inspire directement de celle de Polytechnique ${ }^{75}$.

Durant une grande partie du siècle, peu d'établissements disposent d'un équipement suffisant pour organiser des " exercices pratiques " pour tous les élèves. La fréquentation de l'" école pratique " de la faculté de médecine, instituée en 1797, n'est pas une obligation et seuls y ont accès quelques élèves reçus sur concours. L'École supérieure de pharmacie, sous l'impulsion du pharmacien-chimiste Alexandre Bussy, suivra cet exemple en créant sa propre école pratique en 1831, en plus du stage officinal préalable obligatoire ${ }^{76}$.

72 William Arthur Smeaton, "The Early History of Laboratory Instruction in Chemistry at the École Polytechnique, Paris, and Elsewhere ", Annals of Science, vol. 10, n³, September 1954, p. 224-233.

73 Louis-Jacques Thénard, "Sur la nécessité de réunir la pratique à la théorie de la chimie, pour en faire d'utiles applications aux arts ", Annales de chimie, t. 34, 1800, p. 106.

74 François Leprieur, Les conditions de la constitution d'une discipline scientifique..., op. cit., p. 10.

75 Charles de Comberousse, Histoire de l'École centrale ..., op. cit., 1879, p. 13.

76 Certains pharmaciens, tels Jacques-Philibert Delunel, s'opposent aux chimistes et défendent la préséance de la pratique sur la théorie : J. Simon, "La chimie et la pharmacie en 1800 ", Revue d'histoire de la pharmacie, t. 45, n³30, 2001, p. 175-182. 
Le règlement du 5 février 1841 entérine l'existence de cet enseignement et précise que le concours a pour but de " s'assurer si les élèves ont les connaissances préliminaires indispensables pour profiter de l'enseignement pratique $\aleph^{77}$. Mais, faute de place, les travaux pratiques ne seront effectivement rendus obligatoires qu'à l'installation de l'École dans ses nouveaux locaux, en $1882^{78}$. Au Collège de France, à la faculté des sciences, au Muséum et au Conservatoire des arts et métiers, il n'existe pas de telles organisations et seuls quelques élèves privilégiés, repérés pour leurs aptitudes, sont admis dans le laboratoire plus ou moins équipé du professeur. Au Collège de France, par exemple, Balard et Berthelot dirigent quelques élèves, qu'ils exercent à "l'analyse qualitative et la préparation ainsi que la purification des réactifs " ${ }^{79}$. Au Conservatoire, les cours de Payen, d'Eugène Péligot et de Jean-Baptiste Boussingault, bien que rassemblant plusieurs centaines d'étudiants, dispensent un enseignement où les auditeurs sont "spectateurs d'expériences " sans être "introduits dans le laboratoire " 80 .

Les moyens des établissements publics ne suivent pas la croissance de la population étudiante et les quelques laboratoires privés bien équipés, entretenus par des mécènes et des fortunes personnelles, tels celui de Dumas, du pharmacien-chimiste Jules Théophile Pelouze entre 1838 et 1857, de Deville, de Wurtz, et de Charles Gerhardt en 1851-1852, ne compensent qu'en partie les lacunes du système universitaire ${ }^{81}$. Devant le manque chronique de financements, les professeurs sont contraints de demander une contribution aux élèves, pratique entérinée par le décret impérial du 31 décembre 1864. Dans ce contexte, des modes économiques de manipulations sont proposés aux étudiants ${ }^{82}$. Mais la "noble science " mérite mieux. La logique pédagogique

77 Nicolas-Jean-Baptiste-Gaston Guibourt, Manuel légal des pharmaciens et des élèves en pharmacie, Paris, J.-B. Baillière, 1852, p. 43.

78 Georges Dillemann, La Faculté de pharmacie de Paris, 1882-1982, Paris, Éd. Comarco, 1982, p. 125.

79 Cité par François Leprieur, Les conditions de la constitution d'une discipline scientifique..., op. cit., p. 158.

80 Ibid., p. 121. Les années 1870-1900 marquent un déclin pour l'institution : Claudine Fontanon, André Grelon, "Le Conservatoire des Arts et Métiers. Deux siècles d'histoire ", in Les professeurs du Conservatoire national des arts et métiers. Dictionnaire biographique 1794-1955, t. 1 : A-K, Paris, INRP/CNAM, 1994, p. 23-57.

81 Maurice Crosland, "Research schools of chemistry from Lavoisier to Wurtz ", British Journal for the History of Science, September 2003, vol. 36, n 3, p. 333-361.

82 Henry Violette, Nouvelles manipulations chimiques simplifiées ou Laboratoire économique de l'étudiant,

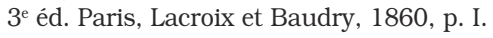


proposée par Lavoisier va être officialisée en 1868. Alerté par divers savants, dont certains cumulent postes universitaires et fonctions politiques (Dumas, Berthelot), ainsi que par des personnalités comme Pasteur, Deville, Frémy ou Wurtz, le ministre de l'Instruction publique Victor Duruy prend conscience que " la meilleure leçon du plus habile professeur ne vaudra pas une expérience que l'auditeur aura faite lui-même ; [car] il faut que les yeux voient et que les mains touchent "83. Ce constat fait écho aux propos émis par Dumas en 1837 : " comment un élève pourra-t-il reconnaître les substances qu'il a vues pendant quelques secondes entre les mains du professeur de chimie ? " ${ }^{84}$ D'où la présence d'une section "Physique et chimie " à l'École pratique des hautes études créée en 1868, ce "Louvre des sciences " que réclamait déjà Hippolyte Fortoul et dont la dimension pratique est inscrite dans son intitulé ${ }^{85}$. La structure ainsi mise en place entérine la division du laboratoire en deux espaces distincts : l'un pour l'enseignement, l'autre pour la recherche. Désormais, les cours en amphithéâtre sont définitivement liés aux laboratoires d'enseignement, qui sont " affectés aux étudiants, aux candidats à la licence, pour que le professeur y répète, ou leur fasse répéter devant lui, les manipulations, les expériences classiques, inséparables de son enseignement "86.

Frémy va s'illustrer au Muséum en appliquant à la lettre cette pédagogie officielle en fin de carrière, après avoir pratiqué un enseignement magistral traditionnel ${ }^{87}$. Lorsqu'il évoque les raisons de la création d'un laboratoire d'enseignement de la chimie, il dénonce les insuffisances du style traditionnel : apprentissage " par cœur " des leçons à travers les ouvrages, examens ne permettant de juger que de la mémoire des élèves ${ }^{88}$. Finie la pédagogie reposant sur les expériences de cours au " caractère brillant ", destinées à " exciter l'éton-

83 Cité par Nicole Hulin, "La chimie au sein des disciplines scientifiques. Perspectives historiques ", Bulletin de l'union des physiciens, $\mathrm{n}^{\circ}$ 815, vol. 93, juin 1999, p. 1018.

84 Jean-Baptiste Dumas, "Rapport adressé au Ministre de l'Instruction Publique ... ", loc. cit., p. 244.

85 Y étaient regroupés à l'origine les laboratoires de chimie de l'École normale supérieure, du Collège de France, du Muséum national d'histoire naturelle, de la faculté des sciences et de la faculté de médecine.

86 Harry W. Paul, "L’idée de recherche dans les facultés des sciences au XIX siècle ", in Christophe Charle, Régine Ferré (dir.), Le personnel de l'enseignement supérieur en France au XIX et XX ${ }^{e}$ siècles, Paris, Éditions du CNRS, 1985, p. 221 (c'est moi qui souligne).

87 P.-P. Dehérain, "Notice nécrologique sur Edmond Frémy ", Revue générale des sciences pures et appliquées, t. 5, 28 février 1894, p. 139-140.

88 Edmond Frémy, "Les laboratoires de chimie ", Encyclopédie chimique, Paris, Dunod, t. 1 (2), 1882, p. 779 . 
nement des auditeurs ". Exit les détails pratiques qui " chargeaient inutilement la mémoire " lors d'un cours " purement théorique ". Pour Frémy, la chimie est une " science outil " dont les applications nécessitent " une longue pratique expérimentale ". Il s'agit de passer des élèves "savants en chimie " à des élèves " réellement chimistes ", en qui puissent se développer "le jugement et l'esprit d'observation ". Les préparateurs ont un rôle essentiel au sein du dispositif. Non seulement ils passent la journée à encadrer les élèves et à assister le professeur, mais ils font des conférences, car "l'enseignement développe les idées et devient souvent l'occasion de conceptions scientifiques originales " ${ }^{89}$. L'enseignement n'est jamais complètement déconnecté de la recherche. C'est donc un enseignement aux techniques de laboratoire qui suit le cours magistral, mais celuici subsiste au motif qu'" il est indispensable, si l'on veut tirer quelque profit des manipulations, de posséder déjà certaines connaissances théoriques " ${ }^{90}$.

Les reconstructions et réaménagements des établissements d'enseignement à partir des années 1880 vont généraliser ce modèle en tenant compte des innovations en matière d'équipements de laboratoire (arrivées de gaz et d'eau courante, " niches " ou " cages évaporatoires " ${ }^{91}$ ). Selon le professeur de l'École centrale Charles de Comberousse, "les exercices pratiques doivent suivre, d'aussi près et en aussi grand nombre que possible, l'exposition des théories ${ }^{92}$. À la fin du siècle, sous la plume du photographe Louis Rousselet, un élève imaginaire de l'École centrale écrit dans une lettre de 1885 qu'en " première année, les manipulations sont simples et consistent dans l'exécution pour chacun de nous des principales réactions et expériences décrites dans le cours de chimie générale. En un mot, c'est l'application pratique de ce cours, qui a pour but de mieux fixer les traits dans notre mémoire, de nous familiariser avec les opérations de laboratoire et de nous donner cette adresse spéciale "93. Les bâtiments reconstruits en 1885 présentent toutes les innovations en matière d'équipements. La surface des laboratoires fait plus que doubler.

89 Ibid., p. 780.

90 Descamps, "Cours de M. Frémy. Utilité de la création d'un laboratoire public de chimie ", Revue des cours scientifiques (Revue rose), 28 mai 1864, p. 350.

91 Il s'agit des ancêtres de nos hottes aspirantes : G. Quesneville, "Rapport sur les laboratoires de chimie en construction dans les Universités de Bonn et de Berlin ", Moniteur scientifique,

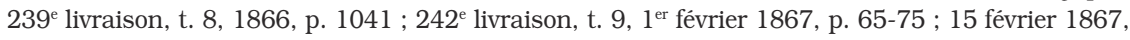
p. 125-130.

92 Charles de Comberousse, Histoire de l'École centrale ..., op. cit., 1879, p. 201.

93 Louis Rousselet, Nos grandes écoles militaires et civiles, Paris, Hachette, 1888, p. 300. 
À l'École des mines, les exercices pratiques prévus par l'ordonnance de 1816 " étaient réputés devoir compléter l'enseignement théorique "94, mais ils ne se généraliseront qu'à la création en 1845 du "bureau des essais " destiné aux analyses particulières, qui libérera le laboratoire ${ }^{95}$.

En 1896, Alexis Lemaistre commente les modifications opérées à la Nouvelle Sorbonne, où l'" on ne se contente plus d'étudier dans les livres, on approfondit, on analyse tout, et la théorie est toujours accompagnée de la démonstration pratique ", en amphithéâtre et dans les quatorze laboratoires de physique et de chimie ${ }^{96}$. Pour le doyen Darboux, l'enseignement de chimie pratique est " coordonné aux cours et conférences de chimie " ${ }^{97}$. En 1901, son successeur Paul Appell salue la création du certificat d'études physiques, chimiques et naturelles ( $\mathrm{PCN})$, enseignement élémentaire obligatoire pour l'inscription dans une faculté de médecine, et note que, sans les exercices pratiques, " les cours magistraux seraient loin de produire tous leurs effets utiles "98.

À l'Institut national agronomique, qui dispose de bâtiments neufs depuis 1890, l'arrêté du $1^{\text {er }}$ avril précise que les chefs de travaux doivent effectuer " des séries de conférences préparatoires aux démonstrations et aux travaux pratiques, ou complémentaires des leçons magistrales "99 (ill. 4). Selon le directeur général des Eaux et forêts Léon Dabat décrivant l'Institut, le " professeur d'analyse et de démonstration chimiques fait marcher parallèlement l'enseignement de l'amphithéâtre avec l'enseignement pratique du laboratoire. Le cours précède toujours les manipulations [...] ; la méthode employée donne d'excellents résultats " ${ }^{100}$.

94 Louis Aguillon, L'École des mines de Paris, Paris, V ${ }^{\text {ve }}$ Ch. Dunod, 1889, p. 100.

95 A. Carnot, "Notice sur l'enseignement de l'École nationale supérieure des mines ", Programmes des cours de l'École nationale supérieure des mines, Paris, Imprimerie nationale, 1900, p. 21 ; "Discours de M. Chesneau prononcé le 13 juin 1914 ", Compte-rendu des fêtes du cinquantenaire de l'association Amicale des élèves et anciens élèves de l'École nationale supérieure des mines, Paris, 1914, p. 75.

96 Alexis Lemaistre, L'Institut de France ..., op. cit., p. 260.

97 Gaston Darboux, "La Faculté des Sciences ", art. cit., p. 54.

98 Paul Appell, L’enseignement supérieur des sciences, Paris, Félix Alcan, 1905, p. 13.

99 Maurice Caullery, "La Faculté des sciences ", in Paul Boyer et al., La vie universitaire à Paris, Paris, A. Colin, 1918, p. 268. On peut voir sur l'élève assis situé à gauche de l'image une inscription et un dessin au dos de sa blouse.

100 Léon Dabat, Rapport du jury international. Groupe I - Éducation et enseignement. Classe 5 : enseignement spécial agricole, Paris, Imprimerie nationale, 1904, p. 96-97. 


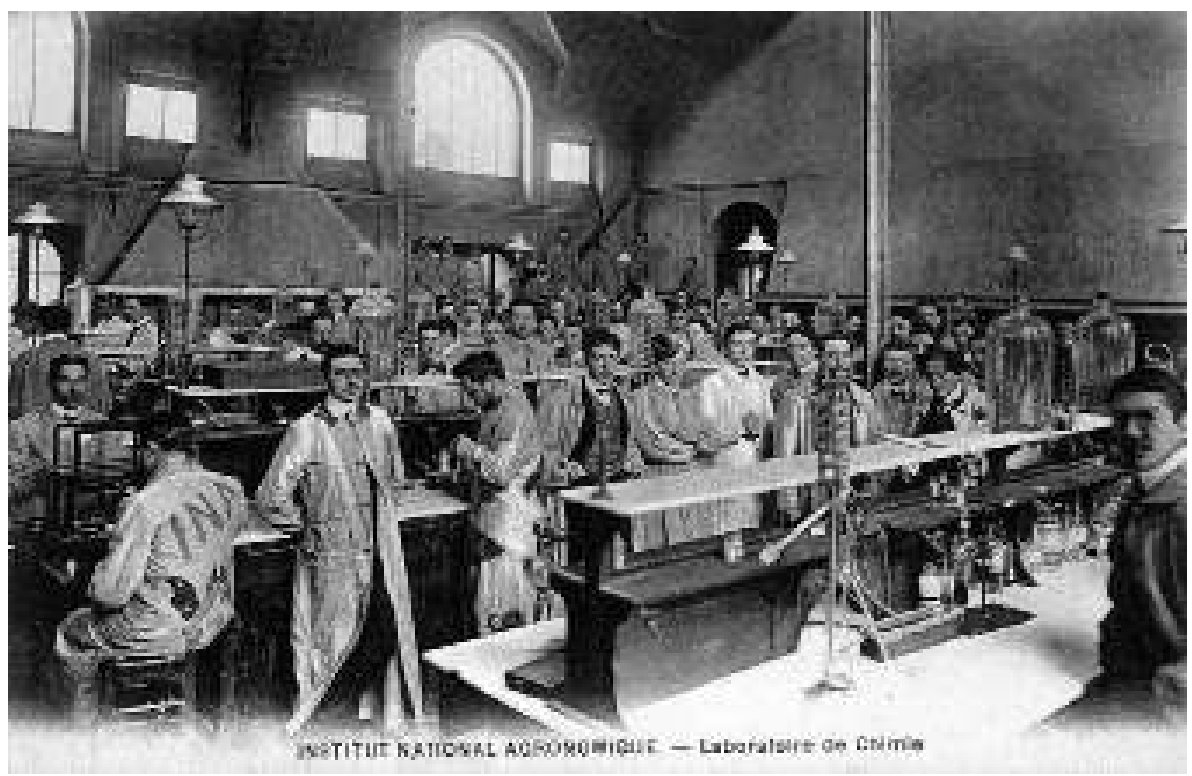

Ill. 4 : laboratoire de chimie de l'institut national agronomique (c. 1900) (Collection particulière)

En 1892, la fermeture quelque peu brutale du laboratoire de Fremy, dans le but d'assainir les finances du Muséum, suscite des contre-mesures. L'enseignement de l'usage des réactifs et de l'analyse constituait la clef de voûte de la pédagogie du professeur. Ce type d'enseignement sera amplement consacré à l'Institut de chimie appliquée, dirigé par Charles Friedel (1832-1899), et à l'École municipale de physique et de chimie industrielles ${ }^{101}$. À l'Institut de chimie appliquée, "l'enseignement pratique qu'on y donne est coordonné aux cours et conférences de la faculté des sciences que les élèves sont tenus de suivre " ${ }^{102}$. Le directeur de l'École municipale de physique et de chimie industrielles, le chimiste et manufacturier Charles Lauth, note la valeur pédagogique de l'analyse chimique, "base de la science " qui s'enseigne les trois années

101 Société de chimie de Paris, De la nécessité de la création d'une grande école de chimie pratique et industrielle, Paris, Paul Dupond, 1891.

102 Paul Appell, L'enseignement supérieur des sciences, op. cit., p. 32. Plusieurs générations d'analystes réputés, tel Gaston Charlot, seront formées à l'Institut de chimie appliquée : R. Rosset, "Gaston Charlot (1904-1994) et le développement de la chimie analytique moderne ", L'Actualité chimique, avril-mai 1995, p. 63-68. 
que durent les études (ill. 5) ${ }^{103}$. Pour Lauth, " la marche des exercices pratiques est commandée naturellement par l'ordre adopté dans le cours du professeur et le développement progressif des sujets qu'il y traite ; il est évident qu'on ne saurait sans inconvénients s'en écarter, ou même les devancer, toute manipulation n'étant en somme que la reproduction des faits ou la confirmation expérimentale des lois exposées dans les leçons orales du maître " ${ }^{104}$.

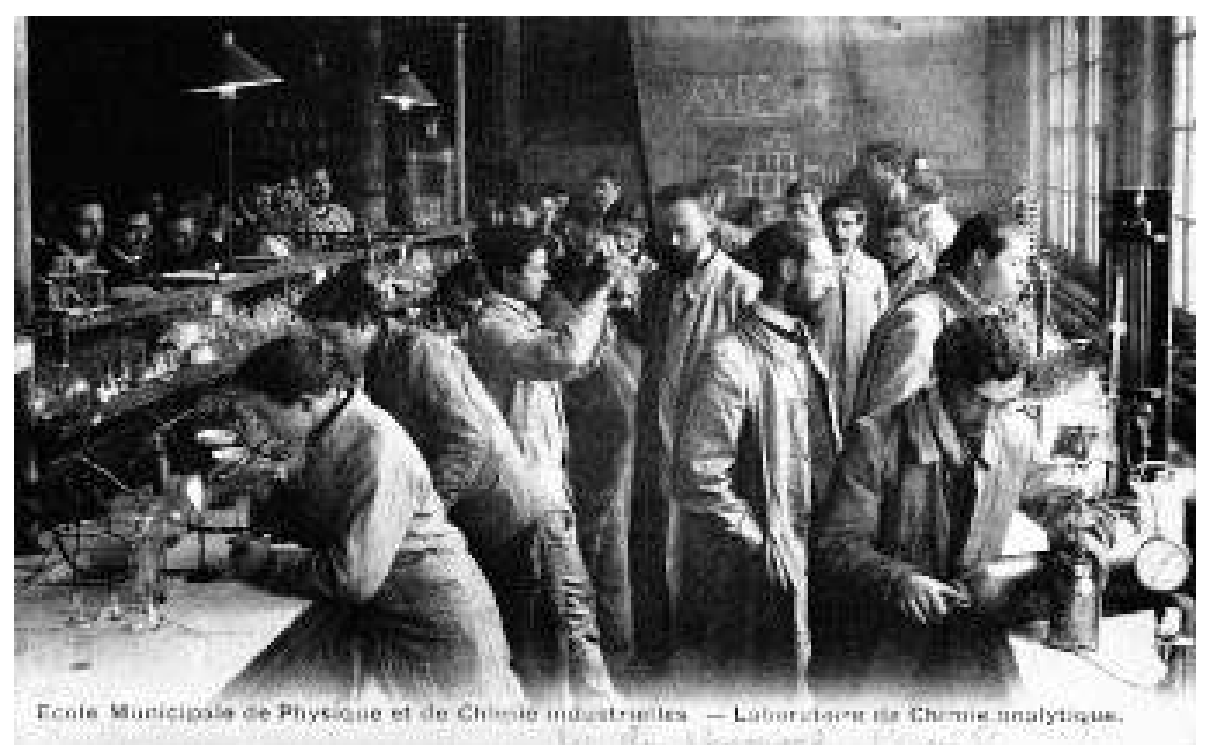

Ill. 5 : laboratoire de chimie analytique de l'école municipale de physique et de chimie industrielles (c. 1900)

(Collection particulière)

Si l'on considère l'ensemble des établissements qui viennent d'être évoqués, un constat s'impose. Le cours magistral plus ou moins agrémenté de " démonstrations expérimentales ", dont l'efficacité s'accroît grâce au rapprochement physique entre l'amphithéâtre et le laboratoire et à l'introduction de différentes techniques visuelles, est le mode d'enseignement le plus répandu

103 On dénombre sur cette image environ 30 personnes, preuve d'une intense activité. On remarque au centre les préparateurs observant les gestes des élèves et, au fond de la salle, quelques élèves discutant d'un montage expérimental dessiné au tableau : Gaston Tissandier, "École de physique et de chimie de la ville de Paris ", La Nature, n 570, 3 mai 1884, p. 376-378.

104 Charles Lauth, Rapport général sur l'historique et le fonctionnement de l'École municipale de physique et de chimie industrielles, Paris, Imprimerie générale, 1900, p. 62 (c'est moi qui souligne). 
durant la plus grande partie du XIX ${ }^{\mathrm{e}}$ siècle. Excepté dans quelques grands établissements (à l'École polytechnique et, dans une moindre mesure, à l'École des mines, à la faculté de médecine, à l'École centrale, à l'École supérieure de pharmacie et à l'École normale supérieure à partir de 1847), c'est seulement à partir des années 1880 que la "méthode analytique " d'apprentissage chère à Lavoisier, celle où les exercices pratiques effectués au laboratoire par les étudiants succèdent au cours magistral, se généralise à tous les établissements et devient une " loi naturelle ". L’amphithéâtre, déjà associé au laboratoire de préparation qui le jouxte, est désormais lié aux laboratoires d'enseignement, largement inspirés des modèles d'outre-Rhin. Et si les bases peuvent s'acquérir en un ou deux ans, la professionnalisation et la création de diplômes de chimiste et d'ingénieur-chimiste ont sensiblement augmenté la durée des études pour la porter progressivement à trois ou quatre ans.

Comment expliquer la longue durée nécessaire à la mise en place des travaux pratiques ? La réponse réside en partie dans la valeur principalement culturelle accordée à l'enseignement de la chimie au XIX ${ }^{\mathrm{e}}$ siècle. Doit-on former des citoyens éclairés, aptes à prendre conscience des enjeux sociaux de la chimie et à les décoder, ou bien des professionnels de l'industrie et de la recherche ? Dans le premier cas, des cours magistraux sont suffisants, tandis que dans le second l'instruction pratique est un passage obligé. Cela explique pourquoi les quelques établissements qui disposent d'écoles pratiques dans la première moitié du siècle sont toutes à caractère professionnel et forment médecins, pharmaciens et ingénieurs. L'industrialisation croissante, offrant de plus en plus d'opportunités de carrière, changera progressivement la nature du cours, l'enseignement de la chimie passant d'un stade où celleci était considérée comme une " science accessoire "à un autre où il devient une formation pour les futurs techniciens et cadres de l'industrie chimique. Cette évolution sera la raison majeure de l'ouverture, en fin de siècle, de centres de formation supplémentaires comme l'École municipale de physique et de chimie industrielles et l'Institut de chimie appliquée. À l'inverse, le Collège de France, le Conservatoire des arts et métiers et le Muséum forment davantage des citoyens que des praticiens et ne délivrent pas de diplômes.

Cette distinction ne permet pourtant pas d'expliquer pourquoi d'autres établissements, tels la faculté des sciences, l'École nationale vétérinaire d'Alfort ou l'Institut national agronomique, bien que menant à des diplômes, ne dispensent pas de séances de travaux pratiques, ou seulement très imparfaitement. 
Dans la perspective globale adoptée par cet article, il apparait que le caractère dissuasif des coûts de l'enseignement de la chimie en personnel et en matériel a également joué un rôle dans la mise en place tardive des travaux pratiques. Les différents équilibres financiers des établissements, souvent fragiles, ne permettaient de maintenir un niveau d'équipement correct que pour un temps limité. Le changement radical de régime, esquissé sous le Second Empire et accentué par la défaite de 1870, n'a été rendu possible que grâce à l'investissement massif consenti sous la III République, le gouvernement répondant ainsi aux revendications des professeurs et industriels portées par des hommes politiques influents, qui étaient parfois eux-mêmes des scientifiques.

À la fin du siècle, les revendications ont porté leurs fruits et les professeurs de chimie ont réussi à stabiliser les conditions matérielles de leur enseignement en trouvant un équilibre entre financements public et privé. Ils disposent pratiquement des mêmes facilités que les luxueux laboratoires d'outre-Rhin. Les laboratoires d'enseignement et de recherche qui se généralisent à partir des années 1880 ne subiront pas d'évolution notable avant la Seconde Guerre mondiale. Le célèbre laboratoire de Pierre et Marie Curie, au carrefour de la science et de l'industrie, est le résultat de cette évolution ${ }^{105}$. L'intervention croissante de l'État stabilise ainsi pour plusieurs décennies un régime de savoir qui est à l'origine de la technoscience du $\mathrm{XX}^{\mathrm{e}}$ siècle ${ }^{106}$. Désormais complété par les travaux pratiques, le cours magistral en est toujours partie intégrante.

Sacha TOMIC

Université Paris 1-Panthéon-Sorbonne, CH2ST - EA 127

s.tomic@free.fr

105 Soraya Boudia, Marie Curie et son laboratoire. Sciences et industries de la radioactivité en France, Paris, Éd. des Archives Contemporaines, 2001.

106 Dominique Pestre, Science, argent et politique. Un essai d'interprétation, Paris, INRA Éditions, 2003. 
